\title{
Surveying the vampire bat (Desmodus rotundus) serum proteome: a resource for identifying immunological proteins and detecting pathogens
}

\author{
Benjamin A. Neely ${ }^{*}$, Michael G. Janech ${ }^{2,3}$, M. Brock Fenton ${ }^{4}$, Nancy B. Simmons ${ }^{5}$, Alison M. \\ Bland $^{2,3}$, Daniel J. Becker $6^{*}$ \\ ${ }^{1}$ Chemical Sciences Division, National Institute of Standards and Technology, NIST Charleston, \\ Charleston, SC, USA \\ 2 Hollings Marine Laboratory, Charleston, SC, USA \\ ${ }^{3}$ Department of Biology, College of Charleston, Charleston, SC, USA \\ ${ }^{4}$ Department of Biology, Western University, London, Ontario, Canada \\ ${ }^{5}$ Department of Mammalogy, Division of Vertebrate Zoology, American Museum of Natural \\ History, New York, NY, USA \\ ${ }^{6}$ Department of Biology, University of Oklahoma, Norman, OK, USA
}

\section{Table of Contents}

Supplemental Figure S1 Relative standard deviation distribution of protein abundance in technical replicate

Supplemental Figure S2 Rh186 viral peptide identification

Supplemental Figure S3 ORF1a viral peptide identification

Supplemental Figure S4 Viral peptide quantities

Supplemental Table S1 Experimental key with sample metadata (xIsx)

Supplemental Table S2 Intermediate data table of vampire bat serum protein ortholog mapping and quantities (xlsx)

Supplemental Table S3 Vampire bat serum protein orthologs and quantities (xIsx)

Supplemental Table S4 NIST 909c human serum protein relative quantities (xIsx)

Supplemental Table S5 Rank comparisons of the vampire bat proteome to the Human Blood Atlas (xlsx)

Supplemental Table S6 Proteins of interest with rank comparisons between vampire bat proteome and the Human Blood Atlas (xlsx)

Supplemental Table S7 Expanded elution group, peptide group, and protein group quantification and associated data (x/sx) 


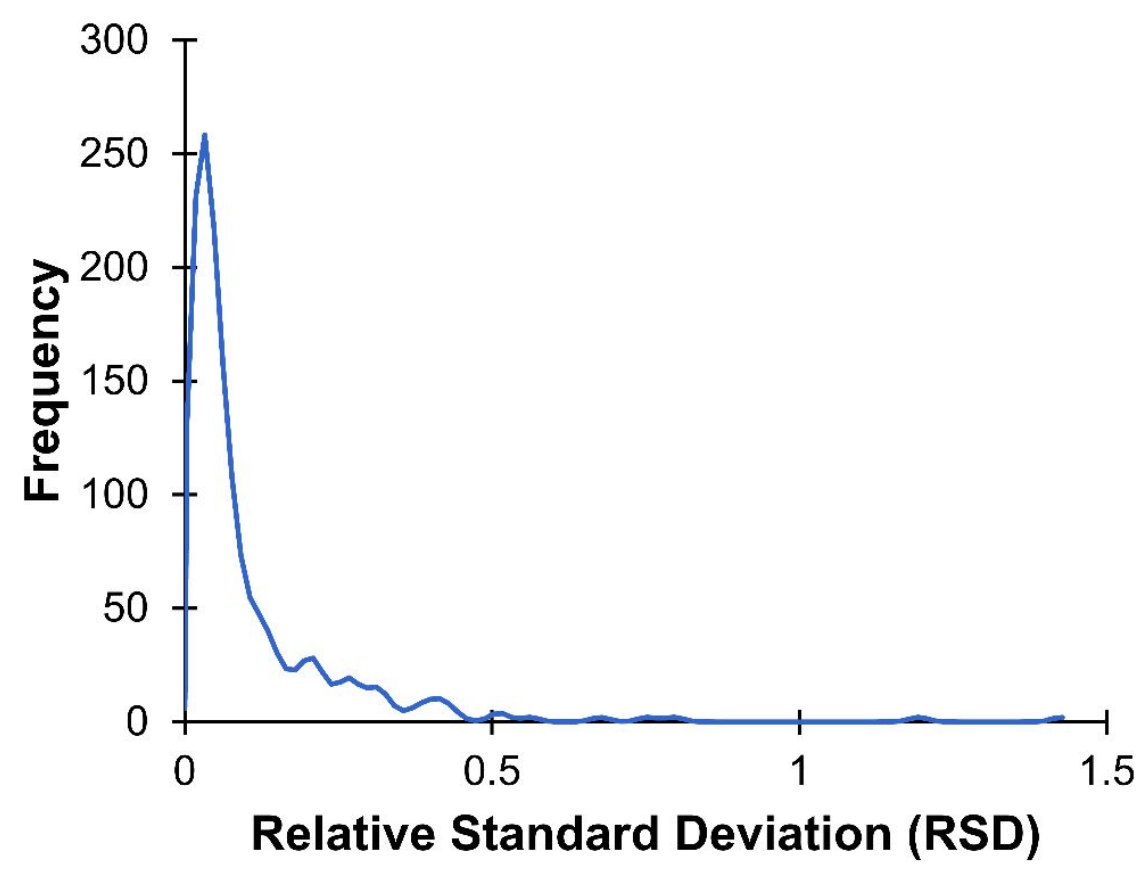

Supplemental Figure S1. Distribution of relative standard deviations in technical replicate. One sample was injected in triplicate to evaluate technical performance of the method. Of the 361 proteins detected across the experiment, between 25 and 30 were filtered out, in total only 322 proteins were identified in all three injections. Using relative abundance from these 322 proteins, relative standard deviation (RSD) was calculated. The average RSD was $10.6 \%$, median RSD was $5.1 \%$, and 270 proteins ( $83.9 \%$ of 322 proteins) had an RSD < $20 \%$. 
A

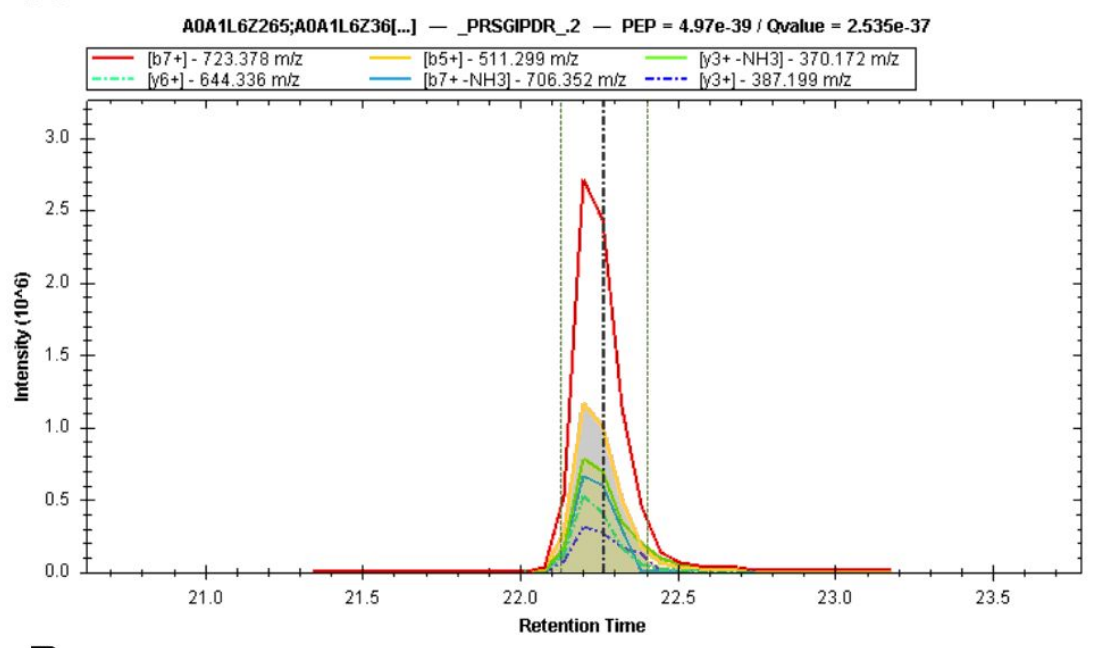

B
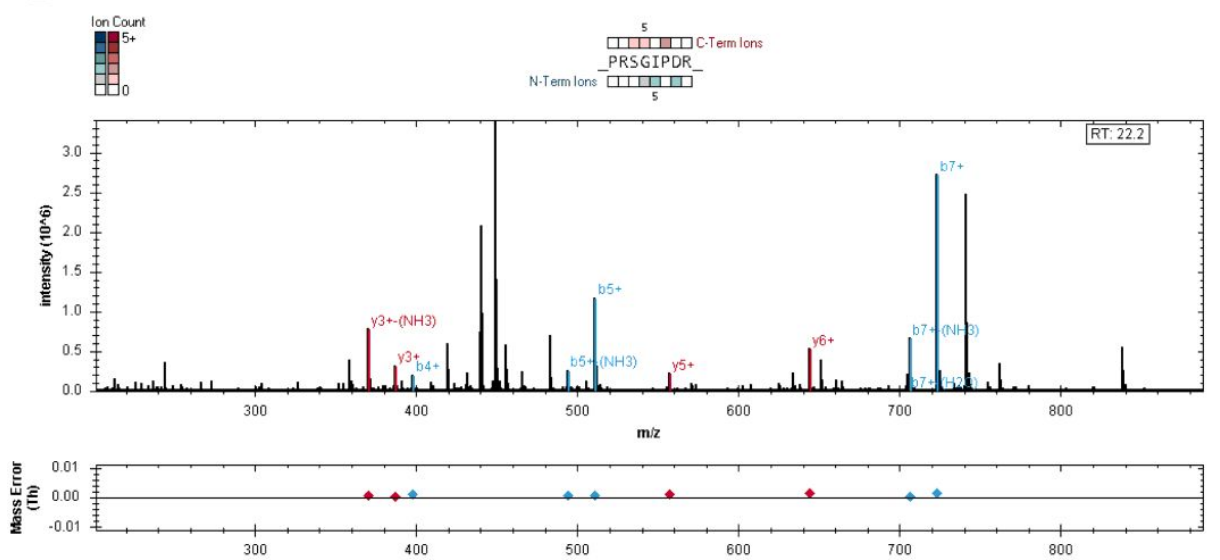

C

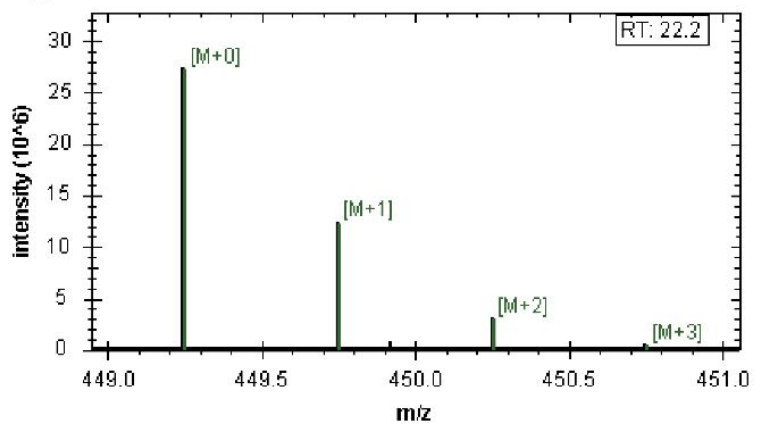

Supplemental Figure S2. Rh186 viral peptide identification. A. Fragmentation ions matching to PRSGIPDR from Rh186 protein in Macacine herpesvirus 3 (i.e., Rhesus cytomegalovirus); UniProt IDs A0A1L6Z265; A0A1L6Z360; I3WF26; Q2FAB1; Q7TFF6. B. Matched b- and y-ions are shown along with mass errors. C. Precursor isotope pattern. Spectra are from Bat_14. 


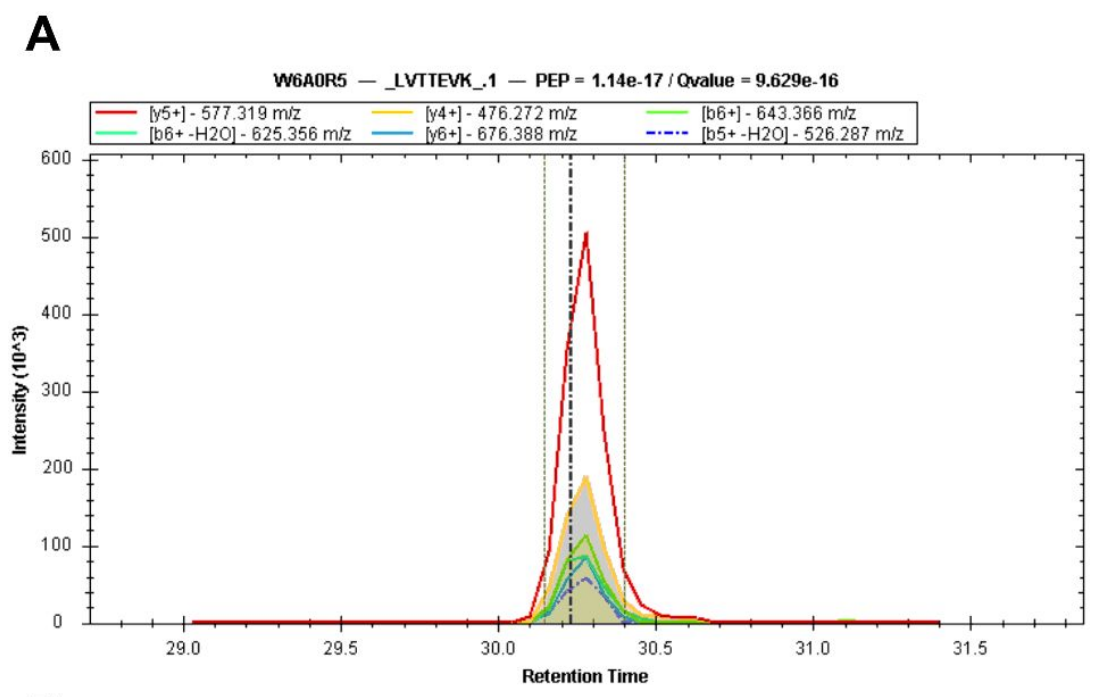

B
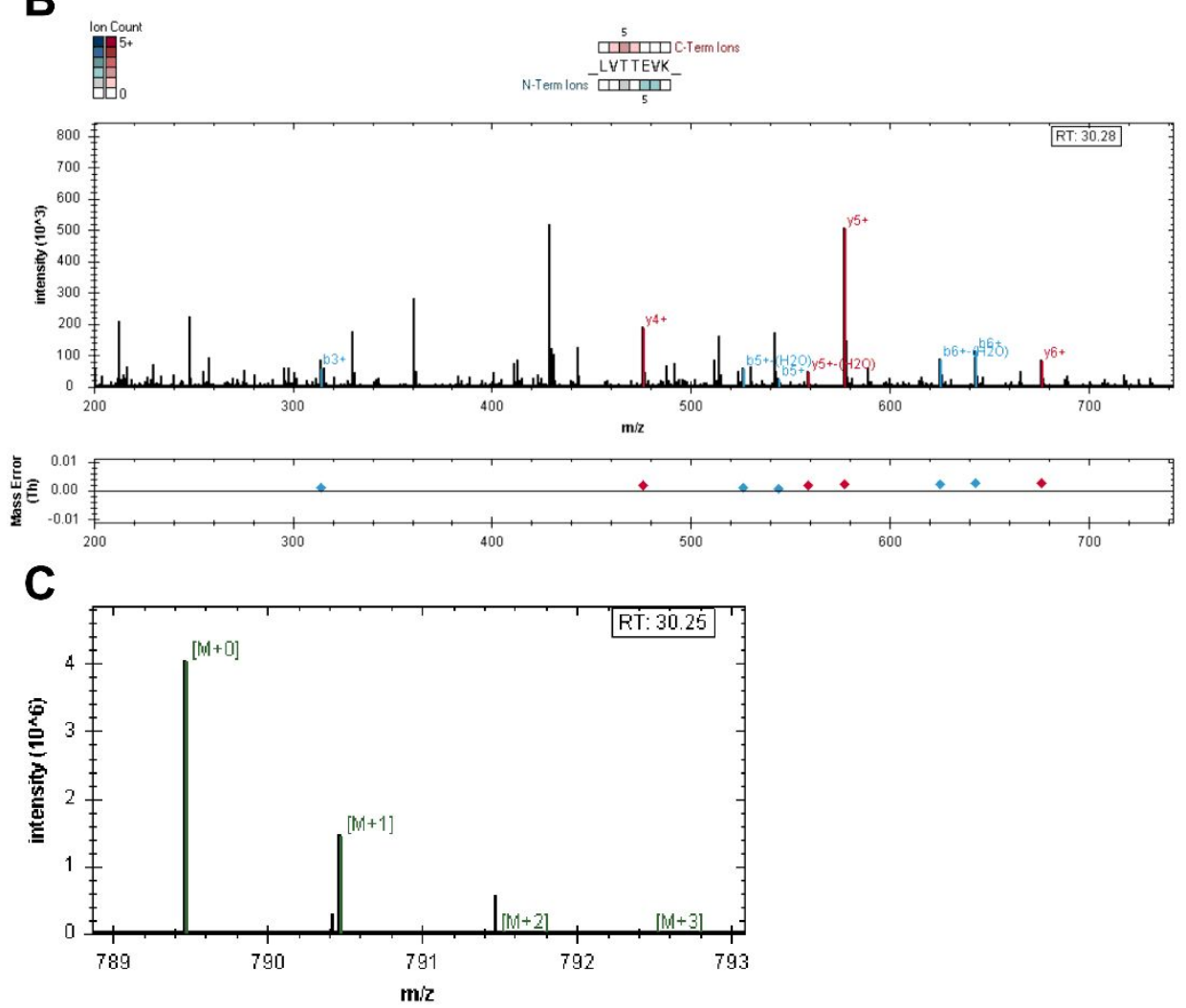

Supplemental Figure S3. ORF1a viral peptide identification. A. Fragmentation ions matching to LVTTEVK from Middle East respiratory syndrome-related coronavirus (MERSCoV), UniProt ID W6A0R5. B. Matched b- and y-ions are shown along with mass errors. C. Precursor isotope pattern. Spectra are from Bat_14. 


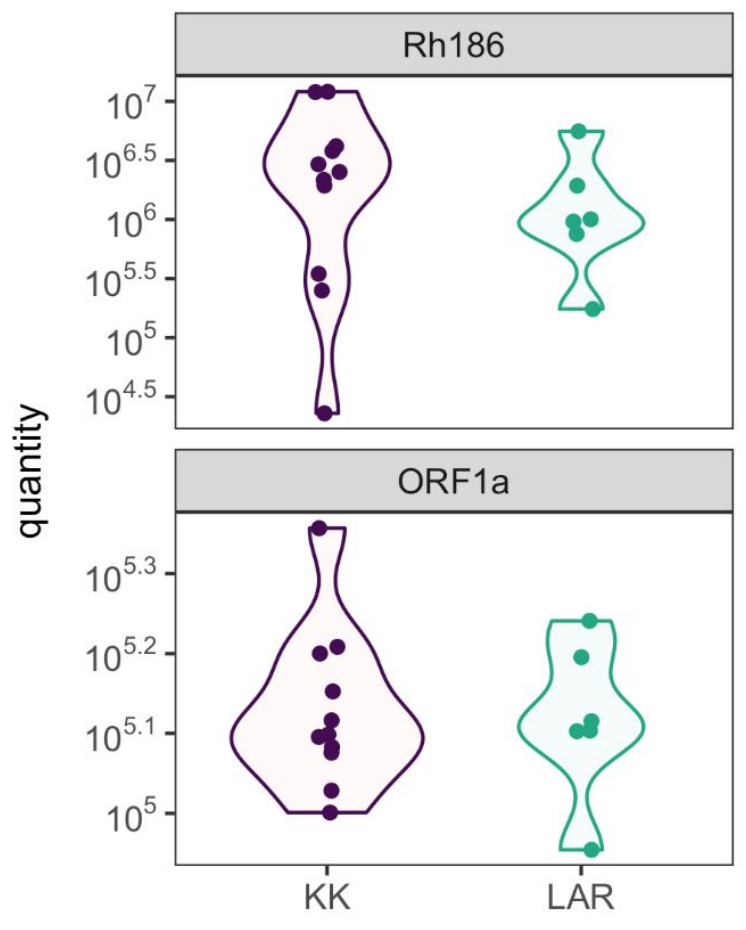

vampire bat sampling site

Supplemental Figure S4. Viral peptide quantities. Violin plots with jittered points for each of the viral peptides (shown in Supplemental Figures S3 and S4) plotted by location. The "quantity" value exported from Spectronaut is the sum of the fragment ion MS2 extracted ion current chromatogram (XIC) areas. These data can be found in "2020-11-23 bat all virii directDIA tryp.sne" (available in PRIDE submission PXD022885). The two sites are two adjacent localities in the Orange Walk District of Belize: Lamanai Archeological Reserve (LAR, 450 hectares) and Ka'Kabish (KK, 45 hectares). 